\title{
ESTILOS DE APRENDIZAJE: ESTRATEGIAS DE ENSEÑANZA EN LUZ
}

\author{
Learning styles: teaching strategies in Universidad del Zulia
}

\section{Engels Ortega*; Ilya Casanova**; Ítala Paredes ${ }^{* * *}$ y Liliana Canquiz ${ }^{* * * *}$}

\section{RESUMEN}

El desarrollo del futuro profesional del diseño gráfico requiere del uso constante de recursos didácticos y financieros para su aprendizaje, producto de un mercado creciente y con mayores exigencias debido al crecimiento global. Esto orienta el camino al uso de estrategias de enseñanza en correspondencia con estilo de aprendizaje del estudiante que fortalezcan sus potencialidades. El objetivo del artículo es evaluar los estilos de aprendizaje presentes en los estudiantes adscritos al programa de Diseño Gráfico de la FADLUZ, para generar estrategias de enseñanza y motivacionales que estimulen su aprendizaje. Se considera una investigación descriptiva y de campo, la muestra estuvo constituida por 59 estudiantes activos e inscritos en las unidades curriculares del Eje Instrumental del Programa de Diseño Gráfico de la FADLUZ a quienes se les administró el Inventario de estilos de aprendizaje de Kolb de 1985 a través de un formulario en Google Form. La mayoría de los estudiantes aprenden mediante la Observación reflexiva (OR) y se inclinan principalmente a dos estilos de aprendizaje el Asimilador con un 51\% seguido del Divergente con un 32\%, se identificó que la tendencia es hacia un aprendizaje más balanceado. Se concluye que la identificación del tipo de estilo de aprendizaje en los estudiantes de Diseño Gráfico, no condiciona hacia un tipo particular de estrategia de enseñanza activa, aun cuando se pueden centrar

\footnotetext{
${ }^{*}$ Magíster en Informática Educativa. Licenciado en Diseño Gráfico. Experiencia docente en pregrado y maestría. Secretario Docente de la Facultad de Arquitectura y Diseño de la Universidad del Zulia. Asesor y diseñador de revistas científicas y libros académicos y páginas Web institucionales. Maracaibo, Venezuela. Correo electrónico: engelsortega@gmail.com

${ }^{* *}$ PhD y Postdoctorado en Ciencias Humanas, Magíster en Educación Mención Planificación Educativa, Licenciada en Bioanálisis. Docente Titular a Dedicación Exclusiva de la Facultad de Odontología de la Universidad del Zulia. Experiencia docente en pregrado y postgrado (especialidad, maestría y doctorado). Autora de capítulos de libro y artículos en revistas arbitradas de alto impacto nacionales e internacionales. Maracaibo, Venezuela. Correo electrónico: iicafe@gmail.com https://scholar.google.es/citations?user=GucTBXUAAAAJ\&hl=es\&safe=strict

${ }^{* * *} \mathrm{PhD}$ en Ciencias Humanas, Magíster en Educación Mención Planificación Educativa, Licenciada en Educación Mención Ciencias Pedagogías Área Tecnología Instruccional. Experiencia docente en pregrado y postgrado a nivel internacional. Docente y coordinadora de la unidad de Diseño Curricular del Instituto de Posgrado de la Universidad Técnica del Norte Ecuador. Autora de capítulos de libro y artículos en revistas arbitradas de alto impacto. Ibarra, Ecuador. Correo electrónico: italmary76@gmail.com

https://scholar.google.com.ec/citations?user=QKwqGacAAAAJ\&hl=es\&safe=strict

${ }^{* * * *} \mathrm{PhD}$ y Postdoctorado en Ciencias Humanas, Magíster en Educación Mención Planificación Educativa, Licenciada en Educación Mención Ciencias Pedagogías Área Tecnología Instruccional. Actualmente docente de la Universidad de la Costa, Colombia. Experiencia docente en pregrado y postgrado. Autora de libros y artículos científicos en revistas arbitradas nacionales e internacionales. Barranquilla, Colombia. Correo electrónico: lcanquiz@ gmail.com https://scholar.google.es/citations?user=OhvN7LkAAAAJ\&hl=es\&safe=strict
} 
Engels Ortega; Ilya Casanova, Ítala Paredes y Liliana Canquiz.

Telos Vol. 21, No. 3 (2019). 710-730

los esfuerzos en el diseño de las actividades, selección del material educativo, forma de presentar la información, creación o no de grupos de trabajo y modificación de los aspectos a evaluar. Es importante que el estudiante identifique su estilo de aprendizaje para fortalecer su proceso de formación. Asimismo, se debe favorecer la motivación que potencie el interés por aprender, sin descuidar el hecho de conocer que estrategias se pueden poner en marcha atendiendo al estilo de aprendizaje.

Palabras clave: formación profesional, estilos de aprendizaje, Inventario de estilos de aprendizaje, estrategias de enseñanza, Diseño Gráfico.

RECIBIDO: Junio 2019

ACEPTADO: Julio 2019

\section{ABSTRACT}

The development of the future professional graphic design requires the constant use of didactic and financial resources for learning, the product of a growing market and with greater demands due to global growth. This directs the way to the use of teaching strategies in correspondence with the style of student learning that strengthen their potentialities. The objective is to evaluate the learning styles present in the students assigned to the Graphic Design program of FADLUZ, to generate teaching and motivational strategies that stimulate their learning. A descriptive and field research was considered, the sample consisted of 59 Students active and enrolled in the curricular units of the Instrumental Axis of the Graphic Design Program of the FADLUZ who were administered the Kolb Learning Styles Inventory of 1985 through a form in Google Form. Most students learn using Reflective Observation (OR) and are inclined primarily to two learning styles. The Assimilator with $51 \%$ followed by Divergent with $32 \%$ identified that the trend is towards a more balanced learning. To conclude that the identification of the type of learning style in Graphic Design students does not lead to a particular type of active teaching strategy, even though efforts can be focused on the design of activities, selection of educational material, presenting information, creating or not working groups and modifying the aspects to be evaluated. It is important for the student to identify their learning style to strengthen their training process. Likewise, motivation should be fostered that fosters the interest to learn, without neglecting the fact of knowing what strategies can be implemented according to the style of learning.

Keywords: vocational training, learning styles, Learning Styles Inventory, teaching strategies, Graphic Design.

\section{Introducción}

La educación superior se ha visto obligada a nuevas formas de actuar y llevar a cabo su tarea educativa, dichas reformas son referente obligatorio de progreso y 


\section{Estilos de aprendizaje: estrategias de enseñanza en LUZ}

desarrollo de sociedades, para abandonar enfoques y renovar algunas facetas en el constante auge de los cambios en el proceso educativo de nuestras universidades. Este cambio educativo compromete a todas las organizaciones a planificar procesos de renovación, cada vez más presentes y comprometidos con las exigencias del mercado laboral, donde la aplicación de novedosas tecnologías es un factor clave en el aumento de la productividad y un factor favorable en la implementación de los alcances del Diseño Gráfico como profesión.

En la actualidad la aplicación de nuevas tecnologías en los diferentes niveles del proceso de diseño, se han transformado y continuamente han influenciado la creación, presentación y comunicación del mismo, sin mencionar el uso de la informática para cada etapa del proceso, de tal manera, que es innegable la necesidad de conocer y manejar sus herramientas para la conclusión exitosa de cualquier proyecto. En este sentido, la universidad debe estar atenta y fortalecer los principios de la profesión, pero sobre todo no caer en la obsolescencia de la tecnología que acompaña la praxis de la profesión.

Lo anterior tiene coincidencia con lo expresado por Paredes et al (2017:652), quienes puntualizan que se requieren de:

"altos niveles de compromiso por parte de los actores del currículo y en especial de la institución educativa lo cual ha de garantizar que los procesos administrativos y académicos sean flexibles, abiertos, proactivos y críticos con una sólida base científico-tecnológica y humana, a fin de contar con estructuras que garanticen la fluidez de los procesos de formación y, sobre todo, la pertinencia social de sus diseños curriculares."

Siendo coherentes con los cambios que se vienen gestando sobre todo en el área tecnológica, el Programa de Diseño Gráfico (PDG) de la Facultad de Arquitectura y Diseño de la Universidad del Zulia (FADLUZ), se enmarca en un modelo educativo cuyos fundamentos epistemológicos dirigen sus esfuerzos a fortalecer la calidad académica con pertinencia social. La institución asume una concepción del hombre como ser que trasciende, con capacidad de respuestas creadoras, generadas a partir de 
Engels Ortega; Ilya Casanova, Ítala Paredes y Liliana Canquiz.

Telos Vol. 21, No. 3 (2019). 710-730

una formación integral que le permite desarrollar las competencias necesarias para enfrentar los problemas del contexto social y lograr el desarrollo humano sustentable.

Con el propósito de una mayor comprensión de los procesos y estructuras inherentes al Diseño Gráfico, es necesario contar con conocimiento teórico - plástico práctico e instrumental que permitan proyectar lo producible y reflexionar sobre lo producido. De allí se puede concluir que, tanto la acción compositiva del diseño como la formación del diseñador, surgen en un plano de integración acotado por ejes donde se conjugan conceptos y destrezas (Facultad de Arquitectura y Diseño [FAD], 2012).

Desde esta perspectiva, el eje de formación instrumental que es donde se centra el abordaje de este estudio: abarca las unidades curriculares donde el estudiante adquiere conocimientos sobre la representación gráfica espacial, así como las habilidades y destrezas necesarias para el uso de instrumentos de dibujo técnico, del computador y el manejo de medios digitales. Sus alcances son similares a los del eje de Formación Expresiva, pero está abocado exclusivamente a los sistemas de representación gráfica, incluyendo el manejo de paquetes informáticos en función del Diseño Gráfico.

Ahora bien, de acuerdo a lo reseñado se comprende que las prácticas que acompañan la formación profesional del diseño, se reflejan en la producción gráfica directa a través de diferentes formatos, sustratos y objetos o publicaciones, sin embargo, es de suponer que carencias en estos recursos, sumado a una infraestructura en sus instituciones poco adecuada para los avances tecnológicos, y la conexión deficiente a redes o sistemas de comunicación, dificulta y desmotiva el aprendizaje, aminorando su productividad.

Es indudable que el contexto actual venezolano implica dificultades, sobre todo en infraestructura y tecnología, lo cual, ha impactado de manera significativa la formación del Diseño Gráfico bajo el modelo educativo actual que demanda el uso de estos recursos y herramientas de manera transversal en los planes de estudio. 
De allí, sea necesario reflexionar, sobre como los estudiantes se conectan con su propio estilo de aprendizaje y logran superar las dificultades al momento de realizar estas experiencias proyectuales. Sistematizar este proceso, puede ser un recurso para el docente que le permita seleccionar estrategias individualizadas para aprovechar cada potencialidad y superar las dificultades del entorno de formación.

Por otra parte, es necesario considerar que el docente cuenta con su propio estilo, evidenciándose en la manera en que él exterioriza la experiencia histórico social del perfil profesional a través de la dinámica del proceso de enseñanza aprendizaje; en el que interactúan variables pedagógicas y personales con un carácter relativamente estable que imprime un sello característico a las relaciones de comunicación que establece en su contexto educativo (Aguilera y Ortiz, 2010).

En vista de las dificultades en los escenarios académicos, se presume que mantener estrategias de enseñanza tradicional, no vinculadas con los estilos de aprendizaje de los estudiantes, supondrá una baja competitividad, poca capacidad y disposición a la actualización de su saber y su oficio, así como escasas herramientas para la producción de proyectos de índole visual y comunicacional, provocando en el estudiante un aprendizaje descontextualizado y mecanizado, que se evidencia al momento de llevarlo a la práctica en el campo laboral, encontrándose el futuro profesional con dudas y vacilaciones al no manejar los principios y los mecanismos para seleccionar los mejores recursos a través de su indagación y actualización constante, lo cual afecta directamente, su producción de propuestas.

La problemática de esta investigación se desarrolla en los espacios de enseñanza como consecuencia de una educación pasiva y centrada en la memoria, muchos estudiantes presentan incluso dificultad para razonar de manera eficaz y al egresar del programa, en muchos casos, presentan dificultades para asumir las responsabilidades correspondientes a la especialidad de sus estudios y al puesto que ocupan, de igual forma se puede observar en ellos la dificultad para realizar tareas trabajando de manera colaborativa. 
Engels Ortega; Ilya Casanova, Ítala Paredes y Liliana Canquiz

Telos Vol. 21, No. 3 (2019). 710-730

Dichos conocimientos en la práctica de la enseñanza en el Diseño Gráfico se han ido construyendo a través de la acción y reflexión, recurriendo a los aportes teóricos disponibles en un momento histórico dado. Este recorrido, acción y reflexión continuos, ha permitido realizar una nueva configuración didáctica para la práctica en el laboratorio, entendiendo como configuración didáctica la manera particular que despliega la acción docente para favorecer los procesos de construcción del conocimiento del profesional en cuestión. En este sentido, la presente investigación se desarrolló con el fin de evaluar los estilos de aprendizaje presentes en los estudiantes adscritos al programa de Diseño Gráfico de la FADLUZ, para generar estrategias de enseñanza y motivacionales que estimulen su aprendizaje.

Esta investigación aun cuando no se enfoca directamente en el docente permite reflexionar sobre las estrategias de enseñanza y como ellas juegan un papel primordial en el proceso de aprehensión de competencias de los estudiantes, optimizando sus labores, bajo un enfoque dinámico y flexible aplicable a las diversas situaciones de aprendizaje y producción gráfica de acuerdo al estilo de aprendizaje de los estudiantes del Programa de Diseño Gráfico de la FADLUZ, y con ello sortear las amenazas que nos es posible eliminar en el contexto actual venezolano.

\section{Una mirada a los Estilos de Aprendizaje}

Aprender se relaciona con la manera que pensamos y esto se vincula con un método o conjunto de estrategias que son propios de cada uno. Aunque estas estrategias concretas varían según lo que queremos aprender, tendemos a desarrollar preferencias globales, esas tendencias a utilizar unas estrategias u otras constituyen nuestro estilo de aprendizaje.

Se puede afirmar que no todos aprendemos de la misma manera, ni a la misma velocidad, aunque contemos con el mismo tiempo, recibamos la misma instrucción e incluso hagamos los mismos ejercicios y prácticas. La motivación, edad y bagaje cultural son factores que influyen en el aprendizaje, pero muchas veces se ha podido 


\section{Estilos de aprendizaje: estrategias de enseñanza en LUZ}

observar a estudiantes que aun cuando cuentan con factores similares, aprenden de manera distinta.

El concepto de Estilos de Aprendizaje ha sido abordado desde perspectivas muy diferentes, no obstante, la mayoría de los autores, se refieren básicamente a rasgos o modos que indican las características y las maneras de aprender de un estudiante. Las distintas teorías han confirmado la diversidad entre los individuos y proponen un camino para mejorar el aprendizaje a través de la conciencia que deben tomar tanto profesores como estudiantes de las peculiaridades diferenciales, es decir, de los Estilos Personales de Aprendizaje. Comprender y considerar el concepto de Estilos de Aprendizaje tanto desde el punto de vista del profesor como de los estudiantes nos dará la posibilidad de actuar para optimizar el aprendizaje.

En este sentido, Alonso et al (1994:48), explican que los Estilos de Aprendizaje son "los rasgos cognitivos, afectivos y fisiológicos que sirven como indicadores relativamente estables, de cómo los discentes perciben, interrelacionan y responden a sus ambientes de aprendizaje".

Es importante señalar que existen diversos modelos que trabajan los estilos de aprendizajes, sin embargo, para este estudio se asumió el modelo de David Kolb que durante su trayectoria académica ha trabajado su teoría experiencial, en la cual se concibe el aprendizaje como un proceso por el cual, se produce un desarrollo del individuo de manera continua basada en la reflexión, que es modificado continuamente por nuevas experiencias.

A partir de la teoría de aprendizaje experiencial, Kolb y Fry (1975: 33-56), define el estilo de aprendizaje como un estado duradero y estable que deriva de configuraciones consistentes de las transiciones entre individuo y su medio ambiente. El autor, propone un modelo de aprendizaje basado en un proceso cíclico de cuatro etapas encadenadas de la siguiente manera:

Experiencia concreta: aprender a través de sentimientos y el uso de los sentidos.

Involucrarse enteramente y sin prejuicios a las situaciones que se le presenten 
Engels Ortega; Ilya Casanova, Ítala Paredes y Liliana Canquiz

Telos Vol. 21, No. 3 (2019). 710-730

Observación y reflexión: aprender observando. Reflexionar acerca de esas experiencias y percibirlas desde varias aproximaciones

Conceptualización abstracta: aprender pensando. El aprendizaje, en esta etapa, comprende el uso de la lógica y de las ideas. Generar conceptos e integrar sus observaciones en teorías lógicamente sólidas

Experimentación activa: aprender haciendo. El aprendizaje, en esta etapa, toma una forma activa. Ser capaz de utilizar eses teorías para tomar decisiones y solucionar problemas.

Según el modelo de Kolb y Fry (1975), para lograr un aprendizaje óptimo, se debe trabajar la información en las cuatro fases anteriormente citadas, constituyendo un ciclo sin fin; por lo tanto, el estudiante actúa, involucrándose totalmente y sin prejuicios en su proceso por capturar experiencias; luego observa y analiza sus experiencias desde diferentes perspectivas, pasando luego a una fase teórica necesaria para adaptar e integrar las observaciones de esa etapa de reflexión; finalmente una etapa pragmática que permite probar ideas, teorías, técnicas nuevas. Este ciclo permanece presente en todo el proceso de aprendizaje del estudiante.

Los estilos de aprendizaje son definidos como algunas capacidades de aprender que se destacan por encima de otras, resultado del aparato hereditario de las experiencias vitales propias y de las exigencias del medio ambiente actual. De lo anterior se desprende que lleguemos a resolver de manera característica los conflictos entre el ser activo y reflexivo, así como entre el ser inmediato y analítico. (Kolb, 1984).

Según Juárez, Rodríguez y Luna (2012), los estilos de aprendizaje son rasgos cognitivos, afectivos y fisiológicos que sirven como indicadores relativamente estables, de cómo los alumnos perciben, interaccionan y responden a sus ambientes de aprendizaje.

Partiendo de esto, la mayor parte de las personas desarrollan estilos de aprendizaje que destacan algunas capacidades para aprender por encima de otras; sin embargo, otras personas desarrollan mentes que sobresalen en la conversación de 


\section{Estilos de aprendizaje: estrategias de enseñanza en LUZ}

hechos dispares en teorías coherentes y, además estas mismas personas son incapaces de deducir hipótesis a partir de su teoría, o no se interesan por hacerlo. Otras son genios lógicos, pero encuentran imposible sumergirse en una experiencia y entregarse a ella (Kolb y Fry, 1975).

En el caso de los docentes, estos siempre develan su estilo de enseñanza, sin embargo, es importante evidenciar de manera concreta las acciones de aprendizaje de los estudiantes, pero contextualizadas dentro de la dinámica de los componentes (objetivos, contenidos, métodos, medios, la evaluación y las formas de organización de la actividad docente), las estrategias didácticas, así como las especificidades del perfil profesional y por el proceso comunicativo que se establece (Aguilera, 2012).

Al respecto Renes y Martínez (2016), manifiestan que las maneras de enseñar de los docentes, así como las formas de aprender de los discentes se hacen visibles diariamente en los centros educativos, resultando imprescindible conocer en profundidad cada una de ellas para dar respuesta a la heterogeneidad educativa.

Por su parte Díaz-Barriga (2010), afirma que es bien cierto que el profesor es el principal agente mediador de los procesos que conducen a los estudiantes a la construcción del conocimiento y a la adquisición de capacidades complejas. Por lo tanto, se espera que los mismos privilegien estrategias didácticas que conduzcan a sus alumnos a la adquisición de habilidades cognitivas de alto nivel, a la interiorización razonada de valores y actitudes, a la apropiación y puesta en práctica de aprendizajes complejos, resultado de su participación activa en ambientes educativos experienciales y situados en contextos reales. Por lo que es necesario identificar los estilos de aprendizaje con los cuales los alumnos acceden al conocimiento, lo procesan y transforman.

\section{Esquema Metodológico}

Según Padrón (2017), el componente metodológico define todo el sistema de operaciones e instrumentaciones que permiten conectar la realidad o el mundo de los hechos (componente empírico) con redes o unidades representacionales de 
Engels Ortega; Ilya Casanova, Ítala Paredes y Liliana Canquiz.

Telos Vol. 21, No. 3 (2019). 710-730

conocimiento supraindividual (componente teórico). El marco metodológico, indica cómo se realizan las secuencias operativas en términos de procesos, tipologías, técnicas e instrumentos empleados para el estudio de las variables a considerar.

Esta investigación tiene un enfoque epistemológico empirista-inductivo, ya que en lo particular está enfocada hacia un caso concreto, independientemente de los conceptos probabilísticos de muestra y población. El espacio de investigación queda identificado con el caso o «escenario». Las generalizaciones (cuando se producen) parten estrictamente de los datos particulares del caso estudiado (Padrón, 2017).

En este caso, se tomó suministró el instrumento a toda la población constituida por los 178 estudiantes activos en el primer período 2016, que estuvieron inscritos en alguna de las unidades curriculares del Eje Instrumental del Programa de Diseño Gráfico de la Facultad de Arquitectura y Diseño de la Universidad del Zulia. El formulario con el Test de Kolb (1985), fue enviado a sus correos electrónicos y se recibieron 73 respuestas del formulario, las mismas fueron depuradas siguiendo el criterio que el instrumento estuviese contestado de manera idónea, de allí que se obtiene una muestra definitiva de 59 estudiantes.

Para la construcción del formulario se utilizó el Inventario de Estilos de aprendizaje de 1985 (Kolb, 1985), que tiene como base el desarrollado por el autor en 1976. El formulario fue adaptado y alojado de forma asíncrona en el servicio Google Forms en el dominio de las cuentas educativas institucionales de la Universidad del Zulia (fad.luz.edu.ve), disponible en línea por invitación. Los resultados se muestran en una base datos automática generada por la aplicación y disponible en una Hoja de Cálculo en Google Docs.

El Inventario de Estilos de Aprendizaje construido inicialmente por Kolb en 1976 toma como base conceptual su modelo de aprendizaje experiencial. Este instrumento evalúa la preferencia por un determinado estilo de aprendizaje, comparando los relativos predominios de una particular modalidad de aprender entre todas las posibles modalidades definidas por el modelo experiencial. 
Es un inventario que puede ser aplicado tanto en forma individual como colectiva, pudiendo incluso en algunos casos ser autoadministrado. Está constituido por 36 palabras, de las cuales 24 están asociadas a cada una de las cuatro fases del ciclo de aprendizaje experiencial, las 12 palabras restantes son incluidas como elementos distractores para controlar la deseabilidad social, de ahí que no son utilizadas para el cómputo final.

Estas palabras evalúan las siguientes cuatro etapas del aprendizaje experiencial: a) Experiencia Concreta (EC), b) Observación Reflexiva (OR), c) Conceptualización Abstracta (CA) y d) Experimentación Activa (EA). Estas etapas (áreas) a su vez conforman las siguientes dimensiones: a) Concreta - Abstracta y b) Actividad Reflexión.

Las respuestas deben ser presentadas por los sujetos ordenando jerárquicamente el grupo de cuatro (4) palabras, según el grado con el cual se percibe a la palabra que mejor caracteriza su estilo individual de aprender asignando puntajes de 1 a 4 , siendo este último para el más característico. El inventario tiene en total nueve (9) grupos.

Kolb (1976), menciona que eligió este tipo de ítem, en la medida que el sujeto en su vida diaria tiende a priorizar las acciones que realiza, de tal manera que esta misma situación se debería presentar cuando se respondiera a la prueba, lo cual hace que al responder el sujeto siempre se enfrente a situaciones en las que debe tomar decisiones. No obstante, en 1985 Kolb realiza una modificación al Test añadiéndole nuevas frases y tal como se hizo referencia, fue seleccionado para elaborar el formulario aplicado a los estudiantes del Programa de Diseño Gráfico participantes (Kolb, 1985).

El formulario construido con base al Inventario de estilos de aprendizaje de Kolb de 1985, contaba con instrucciones donde se les señalaba y ofrecía un ejemplo a los estudiantes de la manera de contestar las 12 frases. Cada frase puede terminarse de cuatro formas distintas, en las instrucciones se les pedía que ordenaran las cuatro opciones de cada frase según pensaran que se ajustaba a su manera de aprender algo nuevo y que trataran de pensar en situaciones recientes en las que se enfrentase con algo nuevo. Las opciones se numeran con un 4 a la terminación que mejor se ajusta a su 
Engels Ortega; Ilya Casanova, Ítala Paredes y Liliana Canquiz.

Telos Vol. 21, No. 3 (2019). 710-730

forma de aprender y con un 1 la que peor se ajusta. Se añadieron dos preguntas antes de iniciar el cuestionario para cumplir con los criterios de inclusión en este estudio, el tipo de respuesta es ipsativa, es decir, no se pueden repetir valores.

Para calcular los puntajes de los modos de aprendizaje y las dimensiones, se elaboraron fórmulas en la hoja de cálculo que permitieron obtener los promedios para ser representados posteriormente en un plano cartesiano de 4 cuadrantes, que definen los estilos de aprendizaje. Se utilizó como herramienta para la representación digital del plano cartesiano, un programa de software libre para graficar funciones matemáticas Graph Versión 4.4.2. De igual manera se calcularon las frecuencias de los estilos de aprendizaje de los estudiantes participantes con el programa estadístico Statistical Analysis System (SAS), versión 2.4 para Windows, y, además, se realizaron gráficos para su mejor comprensión.

\section{Análisis y Discusión de los Resultados}

En el entendido que el Inventario o Test de estilos de aprendizaje de David Kolb, es un instrumento sencillo que permite medir los puntos fuertes y débiles del individuo que aprende, es importante destacar que el test hace énfasis relativo en las cuatro capacidades del individuo para el aprendizaje: experiencia concreta (EC), observación reflexiva (OR), conceptualización abstracta (CA) y experimentación activa (EA).

El inventario proporciona seis puntajes EC, OR, CA y EA, más dos puntajes combinatorios que indican el grado hasta el cual el individuo destaca la abstracción sobre la concreción (CA-EC) y el grado hasta el cual el individuo destaca la experimentación activa sobre la reflexión (EA-OR).

En la Figura 1, se observan la base de datos generada por Google Forms en las cuales se procedió a introducir columnas con las fórmulas que permitieron obtener las puntuaciones tanto de las cuatro modalidades de aprendizaje: EC, OR, CA y EA, como otras operaciones para obtener las puntuaciones finales, que evidenciaron los tipos de aprendizaje dominante: $\mathrm{CA}-\mathrm{EC}=\mathrm{CA} / \mathrm{EC}$ y $\mathrm{EA}-\mathrm{OR}=\mathrm{EA} / \mathrm{OR}$. 
Posterior a esto se procedió a trasladar las puntuaciones al gráfico de representación, como se indicó en la metodología se representaron los datos en un plano cartesiano con la herramienta un programa de software libre funciones matemáticas Graph Versión 4.4.2. Por ello, se hicieron algunas adaptaciones a la representación habitual del Test ajustándolo a las características de la herramienta. Para identificar los planos y los nombres se utilizó un editor de gráficos vectoriales de software libre llamado Inkscape.

Figura 1. Base de Datos generada por el formulario con los puntajes para cada modalidad de aprendizaje y puntajes combinados.

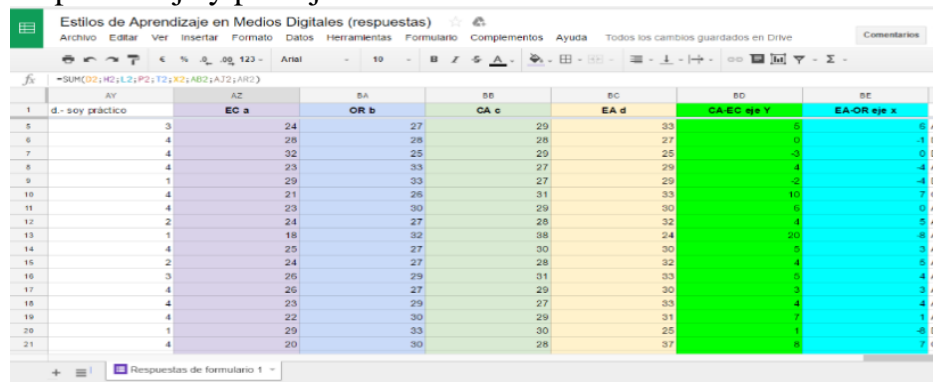

Fuente: Elaboración propia a partir de datos obtenidos en la investigación.

En este estudio se observó un predominio de los estudiantes del Programa de Diseño Gráfico participantes hacia la Observación reflexiva (OR), la cual se puede visualizar en la Figura 2. Al calcular la frecuencia de aparición se obtuvo un porcentaje general de 44,9\% y si consideramos solo las modalidades de Procesamiento es de un $89,8 \%$.

De esta manera se puede afirmar, que la mayoría de los estudiantes participantes entienden el significado de ideas y situaciones con una observación cuidadosa y descripción imparcial de éstas. Disfrutan intuyendo el significado de situaciones e ideas y apreciar diferentes puntos de vista. Les gusta contar con sus propios pensamientos y sentimientos para formar sus opiniones. Son pacientes e imparciales, considerados y 
Engels Ortega; Ilya Casanova, Ítala Paredes y Liliana Canquiz.

Telos Vol. 21, No. 3 (2019). 710-730

muy pensativos. Toman el sentido de una experiencia para reflexionar en ésta y pensar sobre la misma.

En la Figura 2, se puede visualizar también que los estudiantes del Programa de Diseño Gráfico participantes, en segundo término, se inclinan hacia Conceptualización Abstracta (CA) con un porcentaje general de 31,4\% al sólo observar las frecuencias para la modalidad de Percepción, la CA cuenta con un 62,7\%. De manera tal, que otro grupo de los estudiantes participantes usan la lógica, ideas y conceptos; son buenos para la planificación sistemática, manipulación de símbolos abstractos y análisis cuantitativo. Valoran la precisión, el rigor y disciplina de ideas analizadas, así como, la calidad y estética de un sistema conceptual ordenado. Generan información analizando, observando y pensando.

Figura 2. Resultados del Test de Kolb administrado a los estudiantes de Diseño Gráfico de FADLUZ.

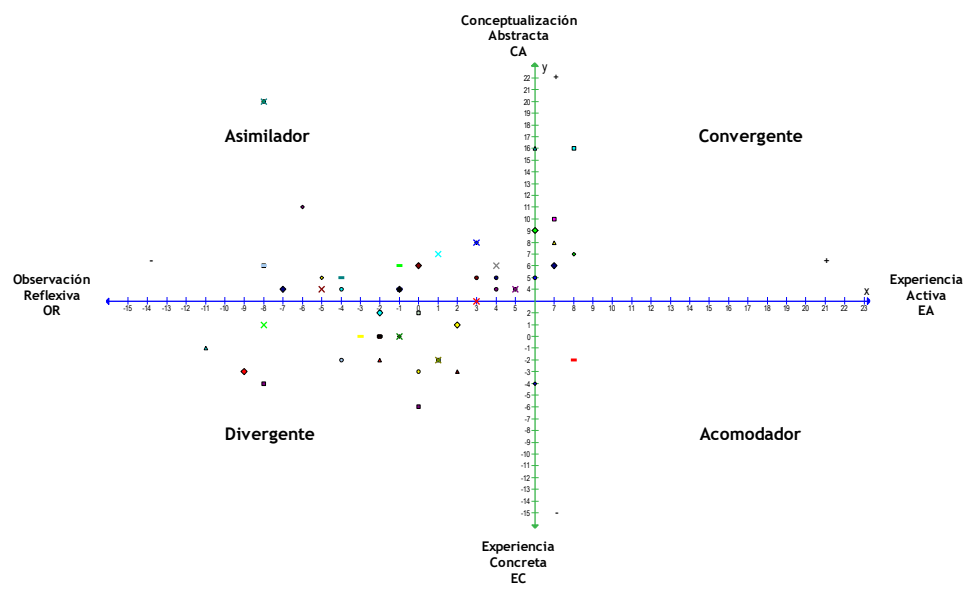

Fuente: Elaboración propia a partir de datos obtenidos en la investigación.

Otro de los elementos a identificar es que mientras más cerca del centro está el punto de intersección de los puntos representados, más balanceado es el estilo de aprendizaje de los estudiantes. Mientras más cerca está de una de las cuatro esquinas, más definidos están en su estilo particular de aprendizaje. En este sentido, se puede 
afirmar que la tendencia en los estudiantes de Diseño Gráfico es hacia estilo de aprendizaje más balanceado, lo cual según Kolb les facilitaría el aprendizaje, independientemente de su estilo de aprendizaje particular.

Cuando se procedió a identificar el estilo de aprendizaje prevalente en los estudiantes de Diseño Gráfico de FADLUZ participantes, como se observa en la Figura 3, la mayoría de ellos se inclina hacia el estilo Asimilador con un 51\%, este estilo de aprendizaje posee un enfoque conciso, lógico y preciso; quienes lo poseen, se destacan por su capacidad para comprender la información, organizándola con un formato claro y lógico. Toman la experiencia de aprendizaje y buscan integrarla a marcos más amplios de teorías abstractas.

Esta gente requiere explicaciones precisas y claras más que prácticas y oportunas. Se sienten más atraídos por las teorías lógicas que por los enfoques prácticos. Del mismo modo, les interesan más las ideas y conceptos abstractos que lo que tenga que ver con las personas. Son teóricos, reflexivos y tienden a ser pacientes, observan, racionalizan y reflexionan. Ven y conceptualizan a fin de construir modelos.

El segundo estilo de aprendizaje que prevalece es Divergente con un 32\%, seguido con porcentajes menores, los estilos de aprendizaje Convergente y Acomodador con un $14 \%$ y $3 \%$ respectivamente.

Los estudiantes con estilo Divergente prefieren observar lo que sucede más que actuar, tienen habilidad para observar un mismo fenómeno desde diversas perspectivas. Del mismo modo, pueden escuchar con amplitud mental, considerando distintos puntos de vista sobre una misma cuestión. Para Kolb y Fry (1975) la denominación de "divergente" se relaciona con las personas que tienden a recabar información y pueden generar una gran cantidad de ideas, a veces originales.

Esto los hace especialmente aptos para situaciones que requieren la generación de ideas, por ejemplo, para procesos de lluvia de ideas. Son personas imaginativas y utilizan esta potencialidad para resolver problemas, cuentan con un pensamiento de tipo inductivo, yendo de lo particular a lo general. Son particularmente sensibles y están 
Engels Ortega; Ilya Casanova, Ítala Paredes y Liliana Canquiz

Telos Vol. 21, No. 3 (2019). 710-730

orientados a la gente. Prefieren trabajar en grupos y se desempeñan bastante bien en el reconocimiento y comprensión de problemas sociales.

Figura 3. Frecuencia por Estilo de Aprendizaje con base en el Modelo de Kolb de los estudiantes de Diseño Gráfico de FADLUZ

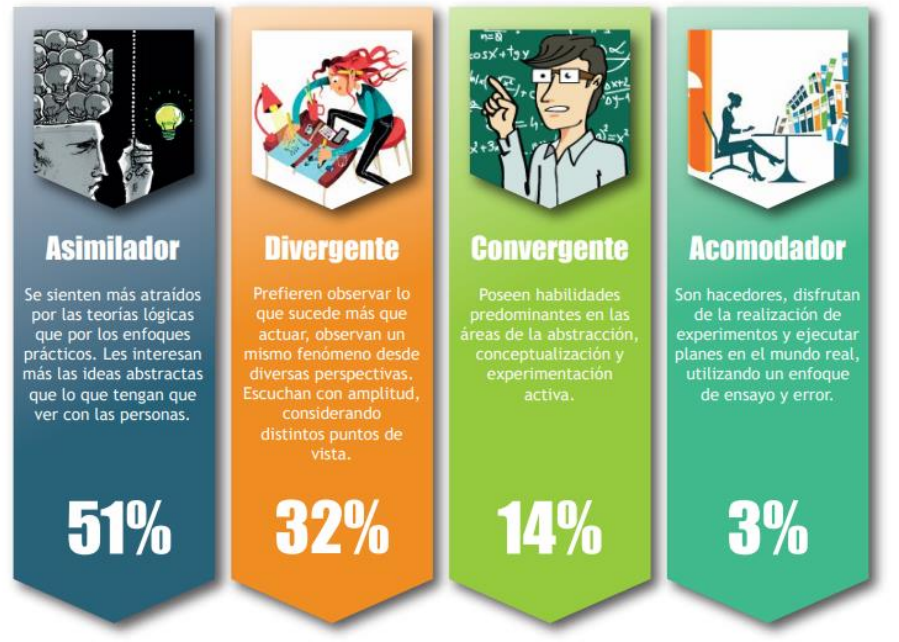

Fuente: Elaboración propia a partir de datos obtenidos en la investigación.

Por otro lado, se coincide con lo expresado por Valdez y Machorro (2014) que existe un denominador común que orienta hacia el manejo de los estilos de aprendizaje y la motivación como aspectos a tomar en cuenta para potenciar el desarrollo del aprendizaje autónomo y significativo en los estudiantes. Ellos puntualizan que es importante tanto para el docente, pero sobre todo para el estudiante, que identifique que situación de aprendizaje prefiere y cual prefiere evitar, sin duda, esto se logra al brindarle la oportunidad de su estilo de aprendizaje.

Incentivar por ejemplo al estudiante hacia un aprendizaje cooperativo pudiese ser una excelente estrategia que permite potenciar la aprehensión del conocimiento al desarrollar las habilidades inherentes a los intereses y potencialidades de cada estilo de 
aprendizaje en conjunto, sin embargo, se debe respetar el aprendizaje autónomo en aquellos estudiantes que así lo prefieran.

Otro de los aspectos que resaltan Valdez y Machorro (2014), es sobre la necesidad de la motivación en el interés por aprender, sin descuidar el hecho de conocer que estrategias que se deben poner en marcha atendiendo al estilo de aprendizaje. Por su parte Díaz y Hernández (2002), sostienen que existe una estrecha relación entre aprendizaje significativo y la motivación; asimismo plantean, que el trabajo motivacional en el aula reside en la habilidad del profesor de poner en contacto a los estudiantes con ideas potentes que permitan vincular estructuras de contenido, clarificar las principales metas de enseñanza y proporcionar por supuesto las bases de aplicación auténticas.

Aun cuando hay una diversidad de estrategias motivacionales que permiten potenciar el aprendizaje de los estudiantes a partir de estrategias de enseñanza activa, no es necesario adjudicarla a ningún estilo de aprendizaje en particular, lo cual refuerza lo expresado por Díaz y Hernández (2002), acerca de que la motivación, depende de la interacción entre el docente y el estudiante pero sobre todo es, un recurso valioso que permite fortalecer el proceso de aprendizaje de los estudiantes, y por ende, la calidad de la educación.

Luego de describir los estilos de aprendizaje de los estudiantes de Diseño Gráfico de FADLUZ participantes, se ha hecho el ejercicio de proponer algunas estrategias sin llegar a establecer una camisa de fuerza que se alejaría en gran medida del propósito de este trabajo que es favorecer el proceso de aprehensión de competencias de los estudiantes, aun cuando es menester recordar, que no se cuenta con un estudiante centrado en una sola forma de aprender, ni con un grupo homogéneo de estudiantes con un mismo estilo de aprendizaje. Es por ello que para su mejor comprensión se han realizado dos infografías (Figuras 4 y 5) donde se exponen que situaciones de aprendizaje que prefieren los estudiantes y aquellas que el docente debe evitar fijando así un precedente importante en la búsqueda de esas estrategias tan necesarias para la comprensión y búsqueda del conocimiento. 
Figura 4. Estrategias de enseñanza activa: situaciones que los estudiantes prefieren y que se deben evitar de acuerdo al estilo de aprendizaje Asimilador y Divergente

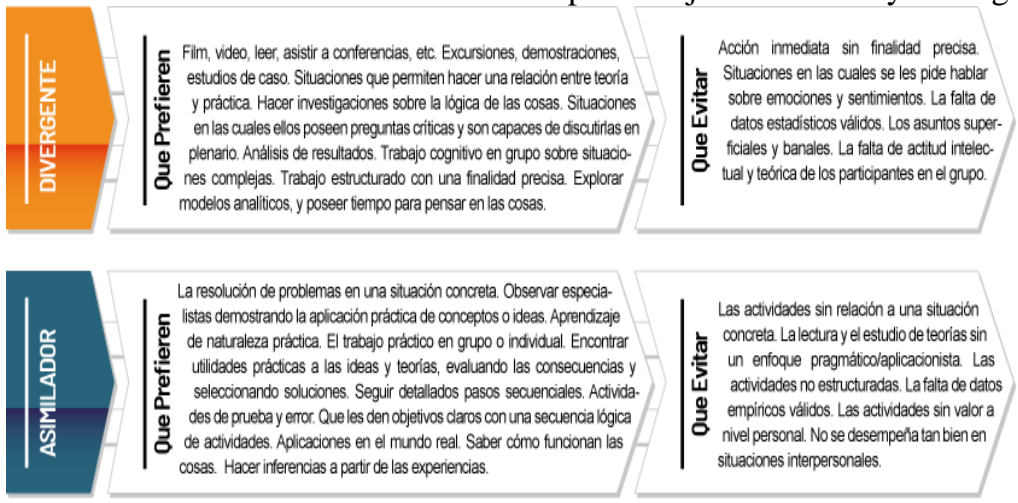

Fuente: Elaboración propia a partir de datos obtenidos en la investigación.

Figura 5. Estrategias de enseñanza activa: situaciones que los estudiantes prefieren y que se deben evitar de acuerdo al estilo de aprendizaje Convergente y Acomodador

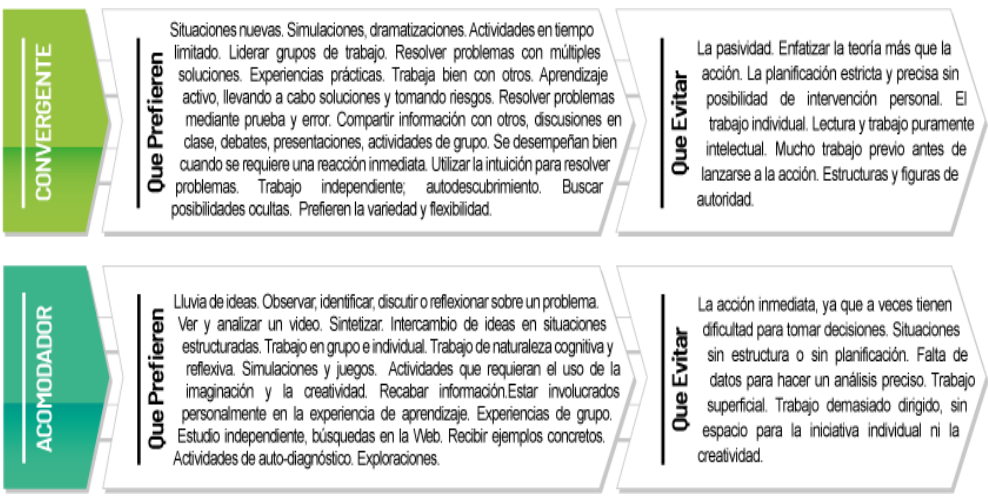

Fuente: Elaboración propia a partir de datos obtenidos en la investigación.

\section{Conclusiones}

El identificar los estilos de aprendizaje en la población estudiantil adscrita a cualquier programa de formación, permite centrar de forma más eficiente los esfuerzos en el diseño de las actividades en el espacio áulico, lo cual involucra la selección de estrategias de aprendizaje, selección del material educativo, formas de presentar la 


\section{Estilos de aprendizaje: estrategias de enseñanza en LUZ}

información, creación o no de grupos de trabajo, fortalecer los aspectos a evaluar, elementos que entre otros, hacen valiosa la construcción y permanente búsqueda de nuevas propuestas didácticas.

La experiencia particular en el Programa de Diseño Gráfico de la FADLUZ, ha permitido la construcción de andamios cognitivos, sustentados en los principios de la profesión, pero cada vez más adaptados a las necesidades particulares de los estudiantes, sin embargo, no se desconoce que facilitarles el desarrollo de competencias, está condicionado por el dinamismo de los cambios contextuales que no siempre ofrece los mejores escenarios, es por ello que las dificultades se superan al enfocarnos en sus potencialidades.

En el caso particular de la población del PDG de la FADLUZ que fue analizada se presentó de manera mayoritaria el estilo de aprendizaje asimilador (51\%), lo cual permitió orientar estrategias con un alto nivel de objetividad y practicidad en los procesos de diseño. Por la naturaleza de este estilo, se manejó el trabajo estructurado, la exploración de modelos analíticos y el estudio de casos, que aumenta la productividad y creatividad de forma sistemática y ordenada, además de la existencia y diversidad de otras estrategias motivacionales que permiten potenciar el aprendizaje de los estudiantes a partir de estrategias de enseñanza activa.

Ahora bien, se debe evitar fortalecer el momento áulico solo para un estilo de aprendizaje prevalente, ya que esta práctica haría perder la intencionalidad de aplicar este formulario de Kolb u otro seleccionado al inicio de un período académico. Es importante que la información aportada sea socializada con el estudiante para que él identifique también su estilo de aprendizaje, de esta manera pueda intencionalmente seleccionar actividades y estrategias que fortalecen su proceso de formación durante toda su vida. Por otro lado, es importante señalar que es importante aplicar estrategias motivacionales que potencie el interés por aprender, lo cual no está ligado a ningún aprendizaje en particular, lo cual abre un abanico de posibilidades para integrar esfuerzos al propiciar el desarrollo de las potencialidades en todo el grupo en formación. 
Engels Ortega; Ilya Casanova, Ítala Paredes y Liliana Canquiz

Telos Vol. 21, No. 3 (2019). 710-730

\section{Referencias Bibliográficas}

Aguilera, Eleanne. (2012). Los Estilos de Enseñanza, Una necesidad para la atención de los Estilos de Aprendizaje en la Educación Universitaria. Revista Estilos de Aprendizaje. Vol. 5, №. 10. España. (Pp. 79-87).

Aguilera, Eleanne y Ortiz, Emilio. (2010). La caracterización de perfiles de estilos de aprendizaje en la educación superior, una visión integradora. Revista Estilos de Aprendizaje. Vol. 3, №. 5. España. (Pp. 26-41).

Alonso, Catalina y Gallego, Domingo. (1994). Estilos individuales de aprendizaje: implicaciones en la conducta vocacional. En: Rivas, F. (ed.), Manual de Asesoramiento y orientación vocacional. España.

Díaz Barriga, Frida y Hernández, Gerardo (2002). Estrategias docentes para un aprendizaje significativo: una interpretación constructivista. McGrawHill. México.

Díaz-Barriga Arceo, Frida. (2010). Los profesores ante las innovaciones curriculares. Revista Iberoamericana de Educación Superior. Vol. I, N ${ }^{\circ}$. México. (Pp. 37-57).

Facultad de Arquitectura y Diseño (FADLUZ). (2012). Diseño Curricular. Universidad del Zulia. Venezuela.

Juárez, Carlos, Rodríguez, Gabriela, y Luna, Elba. (2012). El cuestionario de estilos de aprendizaje CHAEA y la escala de estrategias de aprendizaje ACRA como herramienta potencial para la tutoría académica. Revista Estilos de Aprendizaje. Vol.10 N¹0. España. (Pp.1-31).

Kolb, David y Fry, Ronald. (1975). Toward an applied theory of experiential learning. In Cooper, C. (ed.) Theories of Group Processes. John Wiley \& Song. England.

Kolb, David (1976). The Learning Style Inventory: Technical Manual. McBer. Estados Unidos.

Kolb, David. (1984). Experiential Learning. Experience as the source of learning and development. Prentice-Hall, Inc. Estados Unidos.

Kolb, David (1985). Learning Style Inventory: Self Scoring Test and Interpretation Booklet. McBer. Estados Unidos

Padrón Guillén, José. (2017). Epistemología Evolucionista: Una Visión Integral. GRIN Verlag. Alemania.

Paredes, Itala; Sanservero, Idania; Casanova, Ilya y Avila, Minerva. (2017). Aprendizaje-servicio. Metodología para el desarrollo de competencias integrales en la educación superior. Revista Opción. Año 33, Nº 84 . Venezuela. (Pp. 634-663).

Renes, Paula y Martínez, Pedro. (2016). Una mirada a los estilos de enseñanza en función de los estilos de aprendizaje. Revista de Estilos de Aprendizaje. Vol. 9, Nº. 18 España. (Pp. 224-243). 
Valdez, Verónica y Machorro, Miguel Ángel. (2014). El desarrollo de aprendizaje autónomo a partir de la identificación de los estilos de aprendizaje. Boletín Científico. Publicación Semestral. Vida Científica. $\mathrm{N}^{\circ} 4$ Vol. 2. Universidad Autónoma del Estado de Hidalgo. Secretaría de Desarrollo Internacional. Extraído de: https://repository.uaeh.edu.mx/revistas/index.php/prepa4/article/view/1904 Consulta: $12 / 01 / 2019$ 